\title{
Simulation of Die-swell Flow for Oldroyd-B Model with Feedback Semi-implicit Taylor Galerkin Finite Element Method
}

\author{
Nawalax Thongiub and Vimolrat Ngamaramvaranggul* \\ Department of Mathematics and Computer Science, Faculty of Science, Chulalongkorn University, Bangkok, \\ Thailand \\ * Corresponding author. E-mail: vimolrat.n@chula.ac.th \\ Received: 7 October 2014; Accepted: 11 December 2014; Published online: 2 February 2015 \\ (C) 2015 King Mongkut's University of Technology North Bangkok. All Rights Reserved.
}

\begin{abstract}
This work is focused on creeping die-swell flow for Oldroyd-B fluid in two-dimensional axisymmetric system. The governing equations are solved via a combination of semi-implicit Taylor-Galerkin/pressure-correction finite element method and feedback condition. Some extra techniques for local velocity gradient recovery scheme and streamline-upwind/Petrov-Galerkin method are employed to improve the stability of solutions. For each time step after velocity field is computed, the specific region of die swell jet is adjusted while finite triangle elements in this area are re-meshed. Finally, the benchmark of swelling ratio with other literatures and analytical theory is presented in positive direction.
\end{abstract}

Keywords: Feedback, Semi-implicit Taylor Galerkin finite element method, Die-swell, Oldroyd-B fluid

\section{Introduction}

In this paper, the die-swell problem of Newtonian fluid and polymer melt are set up for simulation of extrudate process in order to survey physical behavior of flow. The free surface shape is computed with streamline prediction method and some solutions inside die near entrance region are picked up and reinforced at inlet boundary condition. This feedback of pressure-driven velocity flow is taken to support the calculation of free surface path. Since fluid motion shown complex deformation when it confronts with intermediate border between stick and slip boundaries, the diameter of extrudate for viscoelastic problem is varied when the property of liquid get more flexibility. The calculation of swelling ratio is computed by the semi-implicit Taylor-Galerkin pressure-correction finite element method (STGFEM) and the treatment of pressuredriven velocity feedback.

For die swell problem of polystyrene samples
[1] in extrusion process, the singularity point was measured by capillary viscometer, which presented the effect of molecular weights on swelling ratio but it got no significance when the aspect ratio L/D (length/diameter) was changed. Consequently, the analytic theory [2] of free surface method under integral transforms was studied for extending stick-slip shape in die. When the flow passes a stick boundary to a free surface, the singular point displays a severe shear stress and steep velocity gradients. To reduce this effect, Okabe [3] has illustrated the semi-radial singularity mapping theory with displaying stress and strain near the singularity. The restriction of analytical solution is not solved widely for various liquids because of the limitation of experiment. As such, so many numerical methods were proposed for appraisal of the surface shape through complex flow. The die-swell or short die problem is a state of art study for wire coating flow [4].

To demonstrate the complicated behavior of

Please cite this article as: N. Thongjub and V. Ngamaramvaranggul, "Simulation of Die-swell Flow for Oldroyd-B Model with Feedback Semi-implicit Taylor Galerkin Finite Element Method," KMUTNB Int $J$ Appl Sci Technol, Vol.8, No.1, pp. 55-63, Jan.-Mar. 2015, http://dx.doi.org/10.14416/j.ijast.2014.12.002 
fluid, the rheological equations and material functions are calculated with the least error approximate solutions such as finite difference (FDM), finite element (FEM) [5-7], and finite volume methods (FVM) [8-11]. These numerical schemes are discretizing techniques that transform continuous equations to a system of linear equations. Some constitutive models of viscoelastic fluid are the form of non-linear partial differential equations that are extremely difficult to solve through analytic methods. Normally, the flow through abrupt surface of die swell case is deformed rapidly to make shear stress grow up to singularity near the die exit. Thus, one numerical research to improve accuracy convergence of solutions was adopted by boundary singularities of integral equation method [12] with free surface scheme for viscous slow flows whilst a technique of mesh refinement [13] on elements at the singularity was applied. Then Crochet and Keunings [14] have considered with slit, circular, and annular dies for Newtonian and Maxwell fluids by a mixed FEM. After exploration of outcome, it showed that the numerical solution gave the result far from real phenomena so Silliman and Scriven [15] have presented a slip condition on die wall to make the result look more real. The same as Phan-Thien [16] who have exhibited the fact of wall slip on extrudate swell and furthermore, the influence of thermal [17] impacted free surface shape. Since the mathematical model of Navier-Stokes and constitutive equations are spatial and time dimensions for multi-variables in terms of velocity, pressure and stresses the couple mode was applied through fractional step method by means of semi-implicit Taylor Galerkin finite element method (STGFEM) $[18,19]$ and the positive result of feedback pressure-driven velocity flow [20] has been presented for the Newtonian fluid through the abrupt 4:1 contraction flow of rounded corner geometry.

In this research, the application of die swell flow on extrusion processes is considered with numerical method of streamline prediction scheme and theoretical approximation. Changing suitable velocity boundary scheme is added to estimate die swell shape as an acceleration to drive solution fast approaching convergence. The numerical solution of STGFEM has been employed to solve the Navier-Stokes equation of Newtonian and Oldroyd-B fluids. Moreover, the stability of approximated solutions is supported by local gradient recovery and the streamline-upwind Petrov/Galerkin techniques under two dimensional axisymmetric isothermal incompressible flow. The solution is recomputed by gradual increase of Weisenberg number (We) to the highest limit. In addition, the pressure-driven velocity flow method is taken to solve the intensive We before the final prediction of swelling ratio is compared with other literatures [21-26].

\section{Governing Equations}

The conservation of mass and momentum under incompressible isothermal viscoelastic flow without gravity is maintained in term of Navier-Stokes equations for two-dimensional axisymmetric system. The dimensionless equations of continuity equation (1) and motion equation (2) are written as shown in [19]:

$$
\nabla \cdot \boldsymbol{U}=0
$$

$\operatorname{Re} \boldsymbol{U}_{\boldsymbol{t}}=\nabla \cdot \boldsymbol{T}-\operatorname{Re} \boldsymbol{U} \cdot \nabla \boldsymbol{U}-\nabla P$

where $\nabla$ is the differential operator, $\boldsymbol{U}$ is velocity vector, $\boldsymbol{U}_{t}$ is time derivative of $\boldsymbol{U}, \mathrm{Re}$ is the non-dimensional Reynolds number that is defined as $\operatorname{Re}=\rho V L / \mu_{0}$ and for creeping flow, $\mathrm{Re}=0$. The extra-stress tensor $\boldsymbol{T}=\boldsymbol{\tau}+2 \mu_{2} \tilde{\boldsymbol{D}}, \boldsymbol{\tau}$ is the extra-stress tensor of the polymeric component, $\boldsymbol{D}$ is the deformation tensor rate or $\tilde{\boldsymbol{D}}=\left[\nabla \boldsymbol{U}+(\nabla \boldsymbol{U})^{T}\right] / 2$, the transpose operator is ()$^{T}$. $P$ is pressure, $\rho$ is the fluid density, $V$ is the characteristic velocity, $L$ is the characteristic length in terms of channel width and $\mu_{0}$ is the zero-shear viscosity for which $\mu_{0}=\mu_{1}+\mu_{2}$ where $\mu_{1}$ is the polymeric viscosity and $\mu_{2}$ is the solvent viscosity. The non-dimensional parameters are $\mu_{0} / \mu_{1}=0.88$ and $\mu_{0} / \mu_{1}=0.12$.

The non-dimensional constitutive equation of a viscoelastic fluid for Oldroyd-B model [27] is

$W e \tau_{t}=2 \mu_{1} \boldsymbol{D}-\boldsymbol{\tau}+W e\left[\tau \cdot \nabla \boldsymbol{U}+(\nabla \boldsymbol{U})^{T} \cdot \boldsymbol{\tau}-\boldsymbol{U} \cdot \nabla \boldsymbol{\tau}\right]$

where $W e$ is the non-dimensional Weissenberg number, $W e=\lambda_{1} V / L, \lambda_{1}$ is the relaxation time.

\section{Numerical Scheme}

The numerical method for this work is based on semiimplicit Taylor Galerkin pressure-correction scheme, which is a fractional step to solve non-linear coupled mode equation on a finite element standard. The 
non-dimensional differential equations (1) - (3) are discretized to the system of linear equations, which are solved via Jacobi iterative algorithm and Cholesky decomposition technique. In addition, the streamline prediction method and alternate velocity boundary condition (Feedback STGFEM) are applied to adjust swelling path precisely.

\subsection{Semi-implicit Taylor-Galerkin pressure-correction finite element method}

The Navier-Stokes equation (2) and constitutive form of Oldroyd-B model were estimated with the calculation of STGFEM, which is split computing to three stages per time step. The time derivative term is expanded with FDM while the spatial component is transformed by the weight residual of standard Galerkin FEM and then the result structure yields partial differential equations (4)-(9).

Step 1a :

$$
\begin{aligned}
& \left(2 \frac{\operatorname{Re}}{\Delta t}\right)\left(\boldsymbol{U}^{n+1 / 2}-\boldsymbol{U}^{n}\right)=-(\operatorname{Re} \boldsymbol{U} \cdot \nabla \boldsymbol{U}-\nabla P)^{n} \\
& +\left[\nabla \cdot\left(\tau+2 \mu_{2} \boldsymbol{D}\right)^{n}\right]+\nabla \cdot \mu_{2}\left(\boldsymbol{D}^{n+1 / 2}-\boldsymbol{D}^{n}\right) \\
& \left(2 \frac{W e}{\Delta t}\right)\left(\tau^{n+1 / 2}-\tau^{n}\right)=\left(2 \mu_{1} \boldsymbol{D}-\tau\right)^{n} \\
& +W e\left[\tau \cdot \nabla U+(\nabla U)^{\mathrm{T}} \cdot \boldsymbol{\tau}-U \cdot \nabla \tau\right]^{n}
\end{aligned}
$$

Step $1 b$ :

$$
\begin{aligned}
& \left(\frac{\operatorname{Re}}{\Delta t}\right)\left(\boldsymbol{U}^{*}-\boldsymbol{U}^{n}\right)=(\nabla \cdot \tau-\operatorname{Re} \boldsymbol{U} \cdot \nabla \boldsymbol{U})^{n+1 / 2} \\
& +\nabla \cdot \mu_{2}\left(\boldsymbol{D}^{*}-\boldsymbol{D}^{n}\right)+\left[\nabla \cdot\left(2 \mu_{2} \boldsymbol{D}\right)-\nabla P\right]^{n} \\
& \left(\frac{W e}{\Delta t}\right)\left(\tau^{n+1}-\tau^{n}\right)=\left(2 \mu_{1} \boldsymbol{D}-\boldsymbol{\tau}\right)^{n+1 / 2} \\
& +W e\left(\tau \cdot \nabla \boldsymbol{U}+(\nabla \boldsymbol{U})^{\mathrm{T}} \cdot \boldsymbol{\tau}-\boldsymbol{U} \cdot \nabla \boldsymbol{\tau}\right)^{n+1 / 2}
\end{aligned}
$$

Step 2 :

$\nabla^{2}\left(P^{n+1}-P^{n}\right)=\left(\frac{2 \mathrm{Re}}{\Delta t}\right) \nabla \boldsymbol{U}^{*}$

Step 3:

$$
\left(2 \frac{\mathrm{Re}}{\Delta t}\right)\left(\boldsymbol{U}^{n+1}-\boldsymbol{U}^{*}\right)=-\left(P^{n+1}-P^{n}\right)
$$

To compute the solution, steps $1 \mathrm{a}, 1 \mathrm{~b}$ and 3 are approximated by Jacobi iterative method whereas Step 2 is determined with Cholesky decomposition scheme before the local velocity gradient recovery and the streamline-upwind Petrov/Galerkin techniques are calculated to stabilize the approximate solution. Finally, free surface is predicted by the streamline prediction method and the alternative velocity boundary technique is considered to improve the swelling ratio by feedback condition or Feedback STGFEM.

\subsection{Theoretical prediction}

The theoretical predictions for adjusting free surface of die-swell in extrudate process are shown in term of swelling ratio $\chi$, which is the proportion between jet radius $\left(\mathrm{R}_{J}\right)$ and tube radius $(\mathrm{R})$, or in the expression $\chi=\frac{\mathrm{R}_{j}}{\mathrm{R}}$. The analytical estimation for elastic fluid under instantaneous elastic strain recovery has been defined by Tanner [21] as equation (10).

$\chi=0.13+\left(1+0.5 S_{\mathrm{r}}^{2}\right)^{\frac{1}{6}}$

Where, recoverable shear $S_{\mathrm{r}}=\left(\frac{N_{1}}{2 \tau_{\mathrm{rz}}}\right), N_{1}$ is the first normal stress difference, $\tau_{r z}$ is the shear stress and ()$_{w}$ is evaluated value at the die wall.

\subsection{Streamline prediction method}

Crochet et al. [28] have stated the boundary conditions (11)-(13) as below. The free surface location of dieswell flow is evaluated from these three conditions by means of streamline prediction method.

$u_{\mathrm{r}} n_{\mathrm{r}}+u_{\mathrm{z}} n_{\mathrm{z}}=0$

$t_{\mathrm{r}} n_{\mathrm{r}}+t_{\mathrm{z}} n_{\mathrm{z}}=S\left(\frac{1}{\rho_{1}}+\frac{1}{\rho_{2}}\right)$

$t_{\mathrm{r}} n_{\mathrm{z}}+t_{\mathrm{z}} n_{\mathrm{r}}=0$

where $u_{\mathrm{r}}$ is radial velocity, $u_{\mathrm{z}}$ is axial velocity, $n_{\mathrm{r}}: n_{\mathrm{z}}$ the unit normal, surface $t_{\mathrm{r}}: t_{\mathrm{z}}$ is force normal, $\rho_{\mathrm{r}}: \rho_{\mathrm{z}}$ is principal radii of curvature and $S$ is surface tension coefficient.

The distance ( $r$ ) from the axis of die symmetry to free surface path is computed by composite Simpson's rule or three-point Newton-Cotes quadrature rule as equation (14). 


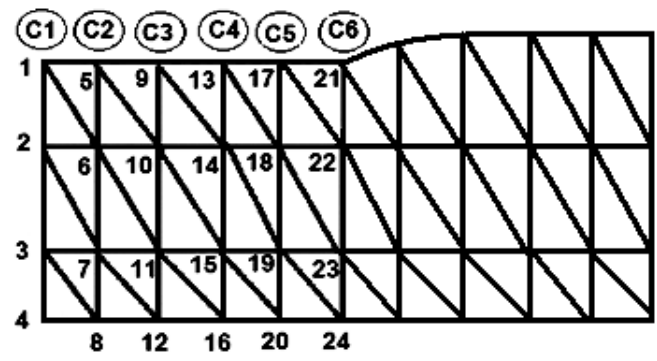

Figure 1: Mesh geometry with node number.

$\mathrm{r}(\mathrm{z})=\mathrm{R}+\int_{z=0}^{\infty} \frac{u_{\mathrm{r}}(\mathrm{z})}{u_{\mathrm{z}}(\mathrm{z})} d \mathrm{z}$

Where $\mathrm{R}$ is die radius.

\subsection{Feedback of pressure-driven velocity flow}

The feedback technique is applied to adjust proper boundary condition at inlet when pressure forces flow to move smoothly through converge solution. For instance of mesh in Figure 1, the possible column can be nodes along $\mathrm{C} 2, \mathrm{C} 3, \mathrm{C} 4$ and $\mathrm{C} 5$. With the selection of $\mathrm{C} 3$, the values of pressure and velocity of nodes 9 , 10,11 and 12 will be the initial condition for nodes 1 , 2, 3 and 4, respectively. Since the computation of large different time step makes solution jump immediately to converge or diverge solution, the differential time step at the beginning is set bigger than the later calculation and then differential time step will be gradually reduced by $10^{-1}$ after the error of outcome was stepped down at the same rate of 0.1 . Every cycle of adjustment for the time step occurred simultaneously with the feedback of the pressure-driven velocity flow to drive the solution in good agreement with the experimental and analytical results.

\section{Problem Specification}

We started from stick-slip problem and then developed to die-swell flow with the same mesh pattern so firstly the designation of mesh style for stick-slip geometry was generated to small sub-cells under 1944 elements and 4033 Nodes as shown in Figure 2 with the smallest size $(\Delta \mathrm{r})$ of finest element to be 0.025 . Before it is run, the boundary conditions are defined as: Poiseuille flow at the inlet, zero

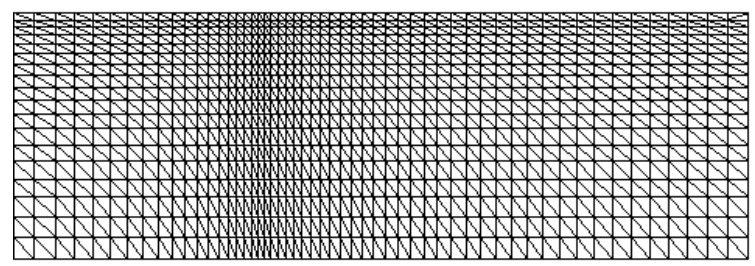

Figure 2: Mesh pattern, 1944 elements, 4033 Nodes.

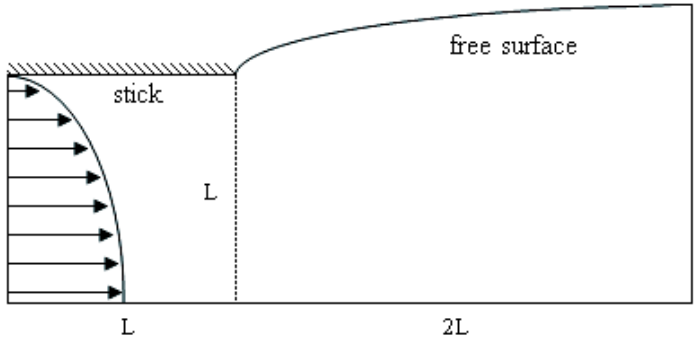

Figure 3: The axisymmetric die-swell flow.

pressure at free surface, null radial velocity for all borders and zero shear stress at symmetry line. The standard die-swell shape is created with $1 \mathrm{~L}$ for entry section and $2 \mathrm{~L}$ for exit portion as displayed in Figure 3 . The same boundary conditions with stick-slip case are set by imposing Poiseuille flow of equation (15) at the inlet which is long enough to complete developing flow that is still retaining parabolic flow pattern. For boundary and initial conditions, at $r=0$ where the flow is symmetric, $u_{\mathrm{r}}=0$ and $\tau_{\mathrm{rz}}=0$. To gradually improve velocity at inlet, the temporary solution was recalculated for feedback condition and the swelling ratio of free surface was calculated after the solution of die-swell problem has been adjusted by feedback condition.

$u_{\mathrm{z}}=1-\mathrm{r}^{2}, u_{\mathrm{r}}=0, \tau_{\mathrm{zz}}=2 W e \mu_{1}\left(\frac{\partial u_{\mathrm{z}}}{\partial \mathrm{r}}\right)^{2}$,

$\tau_{\text {rr }}=0, \tau_{\theta \theta}=0$, and $\tau_{\mathrm{rz}}=\mu_{1} \frac{\partial u_{\mathrm{z}}}{\partial \mathrm{r}}$

\section{Results}

The simulation of die-swell problem is evaluated with STGFEM including feedback condition for fine mesh that has been utilised earlier by Ngamaramvaranggul and Webster [19]. This technique is useful for running high We of Oldroyd-B fluid. For this flow, it is a complex constitutive model so the termination of numerical process is limited at low We. The benchmark of swelling 


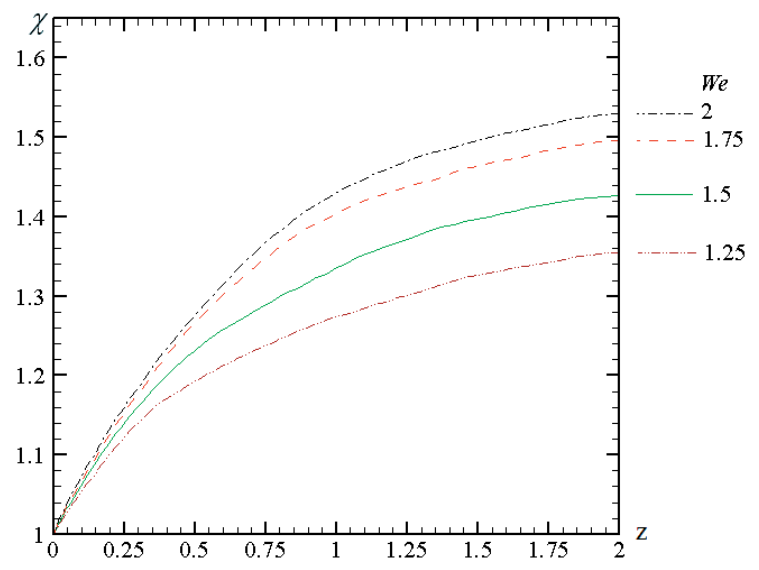

Figure 4: The swelling ratio $(\chi)$ of Oldroyd-B.

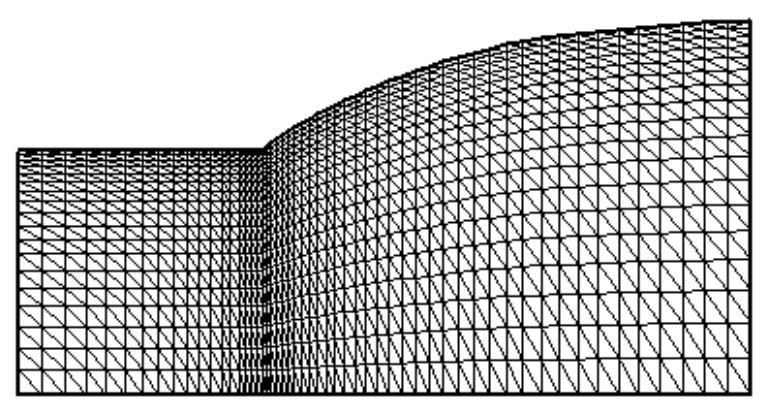

Figure 5: The die swell geometry of $W e=2$.

ratio after feedback treatment is approached to theoretical estimation by Tanner [21] and in addition it spends less time step. For Newtonian fluid, the swelling ratio of Feedback STGFEM is identical to analytical prediction and it is no significant disparity when compared with the solution of Ngamaramvaranggul and Webster [19] but the solution of viscoelastic case for STGFEM with and without Feedback was calculated in terms of swelling ratio $\chi$, normal stress $\left(\tau_{z z}, \tau_{\mathrm{rr}}\right.$, and $\left.\tau_{\theta \theta}\right)$ and shear stress $\tau_{\mathrm{rz}}$ that are identical for both versions but the second invariant II of Feedback STGFEM is higher than STGFEM because the pressure driven velocity adjusted more effect for normal stress in axial direction. The program could run We twice as many feedback condition as no treatment constraint that one can compare this result with the limit of termination at $W e=1$ [19].

In Figure 4, the swelling ratios along the exit of top free surface are varied with $W e$ and all curves are gradually climbed up for the same trend, which

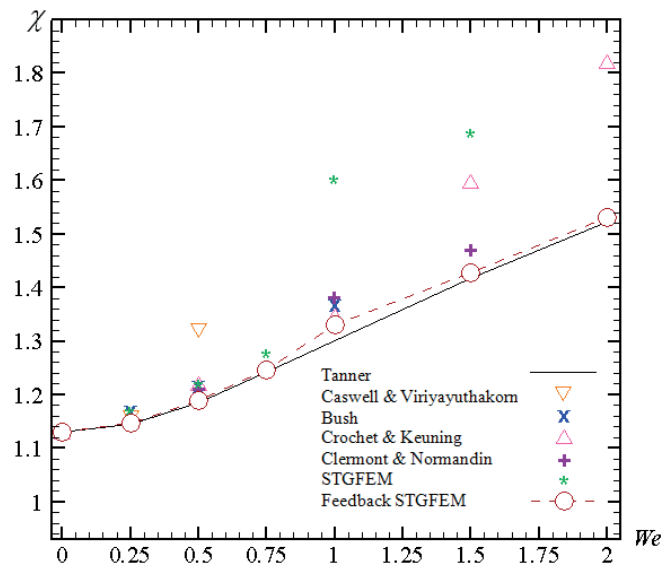

Figure 6: The comparison of $\chi$.

are close to Tanner [21]. To reduce the duplication of many figures, the die swell shape with mesh pattern of highest $W e$ is selected to represent in Figure 5. Since the tendency of swelling ratio for all $W e$ values has the same trend, the swelling ratio increases when $W e$ is higher as seen in Table 1.

Table 1: Benchmark of swelling ratio for various $W e$

\begin{tabular}{|l|c|c|c|c|c|c|c|}
\hline $\boldsymbol{W e}$ & $\mathbf{0}$ & $\mathbf{0 . 2 5}$ & $\mathbf{0 . 5}$ & $\mathbf{0 . 7 5}$ & $\mathbf{1}$ & $\mathbf{1 . 5}$ & $\mathbf{2}$ \\
\hline S1 & 1.131 & 1.146 & 1.186 & 1.242 & 1.301 & 1.417 & 1.523 \\
\hline S2 & 1.131 & 1.161 & 1.325 & - & - & - & - \\
\hline S3 & 1.134 & 1.171 & 1.219 & - & 1.371 & - & - \\
\hline S4 & 1.126 & 1.147 & 1.217 & - & 1.343 & 1.595 & 1.817 \\
\hline S5 & - & - & 1.210 & - & 1.380 & 1.470 & 1.530 \\
\hline S6 & 1.130 & 1.162 & 1.212 & 1.268 & 1.593 & 1.680 & - \\
\hline S7* & 1.131 & 1.148 & 1.190 & 1.247 & 1.330 & 1.427 & 1.530 \\
\hline
\end{tabular}

where

$\mathrm{S} 1=$ Swelling ratio of Tanner [21]

S2 = Swelling ratio of Caswell \& Viriyayuthakorn [22]

S3 = Swelling ratio of Bush et al. [23,24]

S4 = Swelling ratio of Crochet \& Keuning [25]

S5 = Swelling ratio of Clermont \& Normandin [26]

S6 = Swelling ratio of STGFEM [19]

$\mathrm{S} 7 *=$ Swelling ratio of Feedback STGFEM

As per the following result of Feedback STGFEM, the capability of scheme can access the largest value $W e=2$ and gives the best values of swelling ratio when compared with Tanner [21] as illustrated in Table 1 and Figure 6 . Within the range of $0 \leq W e \leq 2$, all values of pressure, stresses, shear rate and II bear the same inclination that tend to rise up implying that these worth proportion to $W e$ as shown in Table 2. With the increase of $W e$, the pressure dragged fluid away to 


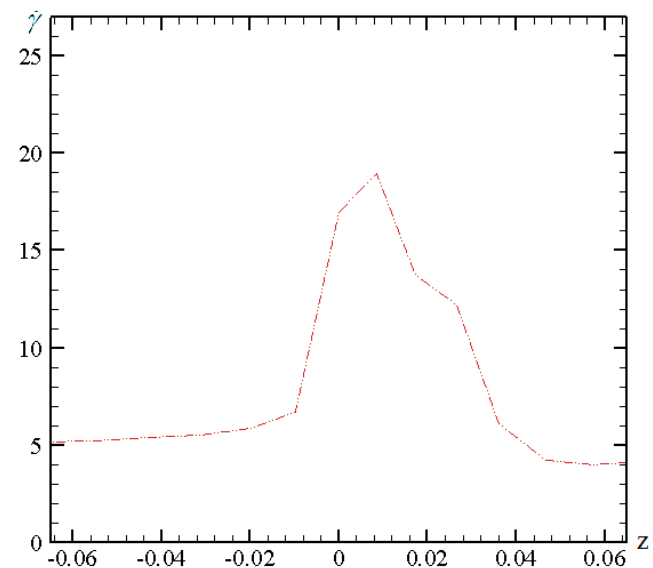

(a) $\mathrm{We}=1.25$

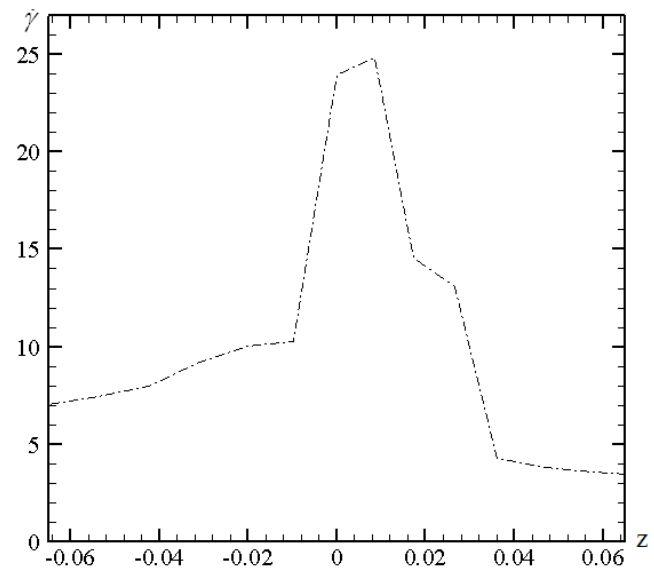

(b) $W e=2$

Figure 7: $\dot{\gamma}$ along the wall.

outside with stronger force and resulted in high pressure drop between entry and die exit.

Since the behavior of second invariant (II) and shear rate $(\dot{\gamma})$ of Oldroyd-B fluid for We between 0 and 2 is similar to normal distribution, a few instances figures of maximum shear rate for $W e=1.25$ and $W e=2$ in Figure 7 are presented in order to avoid duplication. $\dot{\gamma}$ is steady near 5 from $z=-1$ to -0.01 and then it overshoots to the zenith value at exit die $(z=0)$ or the singularity point so these curves show the supreme shear rate impact at the singularity location before it sharply reduces to constant value from $z=0.04$ to $z=2$. Once the fluid passes exit die, the peaks values of shear rate rose immediately and the rate of growth for peak value between $\dot{\gamma}$ and $W e$ is in linear progression.

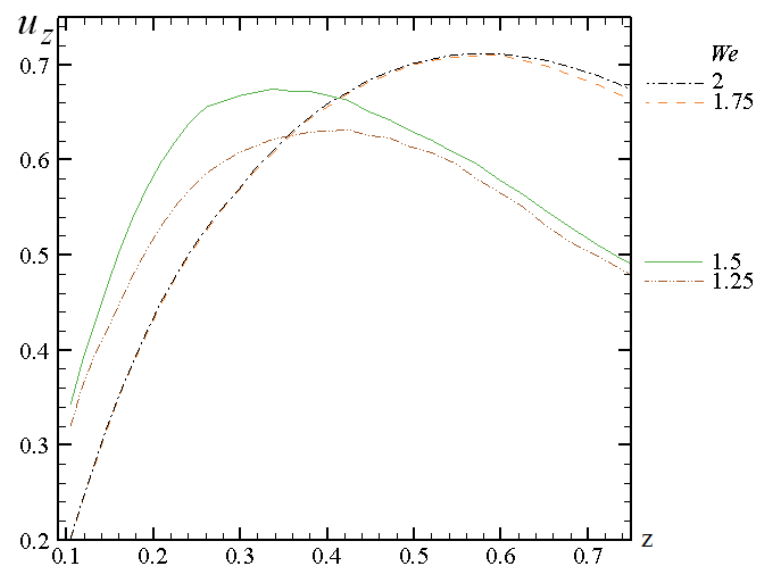

Figure 8: $u_{\mathrm{z}}$ along the wall.

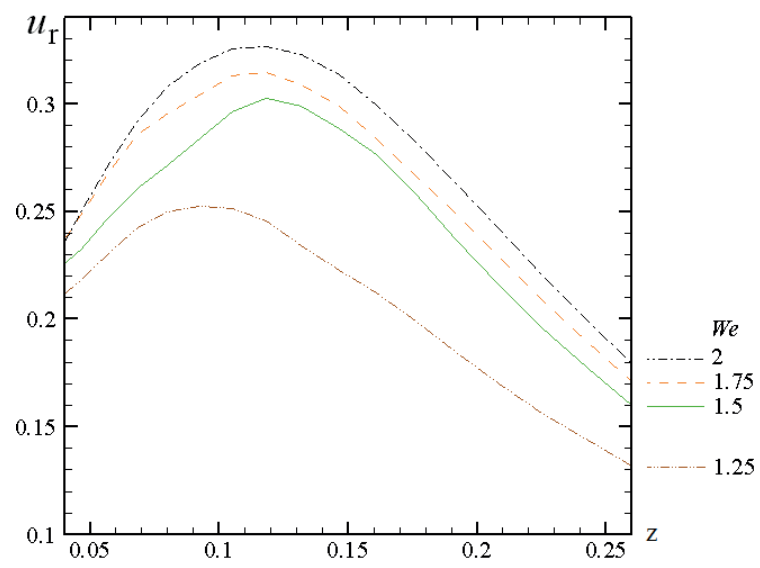

Figure 9: $u_{\mathrm{r}}$ along the wall.

Table 2: The peak values of pressure, stresses, shear rate and $\mathrm{II}$ at $0 \leq W e \leq 2$

\begin{tabular}{|c|c|c|c|c|c|}
\hline $\boldsymbol{W e}$ & $\boldsymbol{P}$ & $\boldsymbol{\tau}_{\text {zz }}$ & $\boldsymbol{\tau}_{\text {rz }}$ & $\dot{\boldsymbol{\gamma}}$ & II \\
\hline 0 & 4.94 & 12.10 & 1.06 & 10.96 & 28.57 \\
\hline 0.25 & 7.24 & 12.81 & 1.95 & 13.58 & 46.08 \\
\hline 0.5 & 16.29 & 13.07 & 3.85 & 15.18 & 60.75 \\
\hline 0.75 & 23.57 & 13.52 & 6.09 & 16.61 & 71.66 \\
\hline 1.0 & 30.31 & 13.70 & 6.83 & 16.93 & 71.66 \\
\hline 1.25 & 32.39 & 14.17 & 7.72 & 18.94 & 89.72 \\
\hline 1.5 & 33.36 & 14.74 & 8.05 & 20.37 & 104.07 \\
\hline 1.75 & 34.20 & 17.94 & 10.16 & 22.83 & 130.26 \\
\hline 2.0 & 34.87 & 18.76 & 10.91 & 24.82 & 153.99 \\
\hline
\end{tabular}

The velocity profiles for die swell in axial direction and radial axis along the wall are shown in Figure 8 and Figure 9, respectively. 


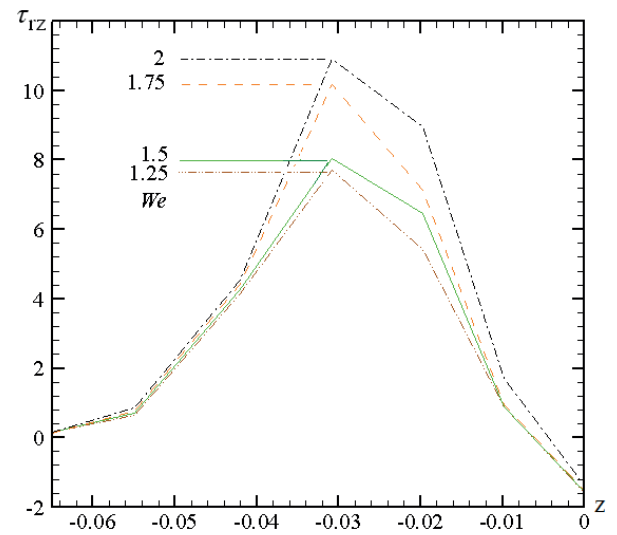

Figure 10: $\tau_{\mathrm{rz}}$ along the wall.

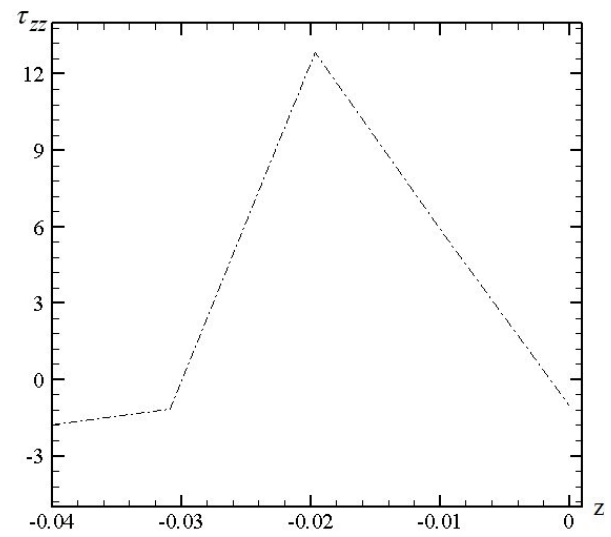

Figure 11: $\tau_{\mathrm{zz}}$ along the wall at $W e=2$.

The peak of $u_{z}$ increases due to the enlargement of $W e$ same as for $u_{r}$ with maxima placed near $\mathrm{z}=0.1$. According to the viscoelastic fluids, the properties of both viscous and elastic flows are noticed in mode of relaxation time representing the memory of stress behavior. The more relaxation time or larger $W e$ is concerned, the more retention of flow path is observed affecting the peak relocation of axial velocity and shift-aways. When $W e=1.25$ and 1.5 , the settle points are placed near $\mathrm{z}=0.35$ while the place of peak shifts to $\mathrm{z}=0.6$ at $W e=1.75$ and 2 as high viscoelatic fluid gathers up velocity and force.

The peak values of shear stress $\tau_{\mathrm{rz}}$ for all $W e$ lie near die exit $(-0.03 \leq z \leq-0.02)$ even if $W e$ is increased but the location of zenith values is the same as seen in Figure 10. The shear stress grows up and then drops near $\mathrm{z}=-0.25$ and the peak values shoot up for higher $W e$ values. To reduce similar graphs of other $W e$, only one normal stress $\tau_{z z}$ along upper die wall is presented in Figure 11, which reveals the highest value at $\mathrm{z}=-0.2$ and the peak values of these normal stresses for various $W e$ are given in Table 2.

Figure 12 displays the line contour of Oldroyd-B fluid at $W e=2$ with conspicuously swelling geometry. The maximum value of velocity $u_{\mathrm{z}}$ still lies on symmetry line as Figure 12(a) but the maximum value of $u_{r}$ is located at exit point as seen in Figure 12(b) whilst Figure 12(c) demonstrates pressure contour that is supreme at entrance and vanished downstream the die region. The maxima of $\tau_{\mathrm{rz}}, \tau_{\mathrm{zz}}$ and $\tau_{\mathrm{rr}}$ at exit die are displayed in Figure 12(d) - Figure 12(f) respectively whilst for $\tau_{\theta \theta}$ the maxima appear at swell area as can be seen in Figure 12(g).

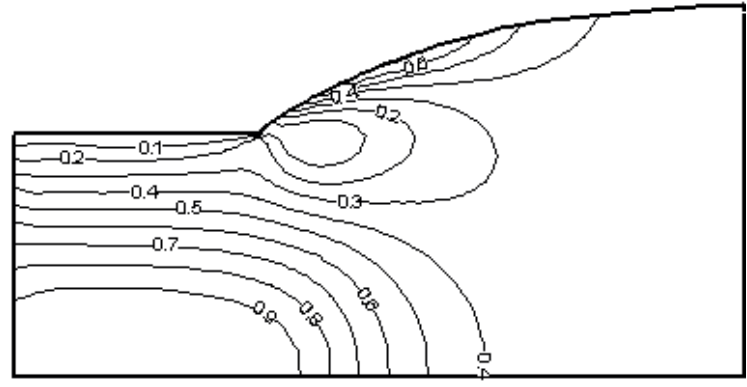

(a) $u_{z}$

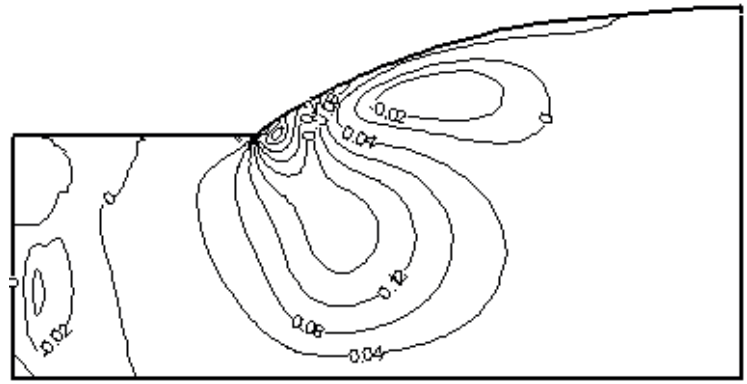

(b) $u_{\mathrm{r}}$

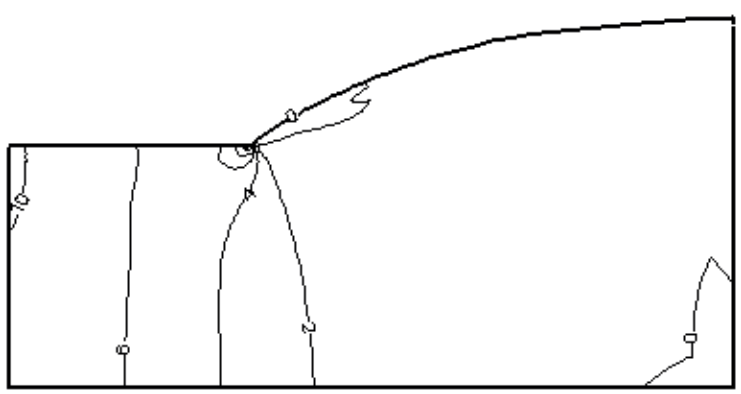

(c) $P$

Figure 12: Line contour of Oldroyd-B fluid at $W e=2$. 


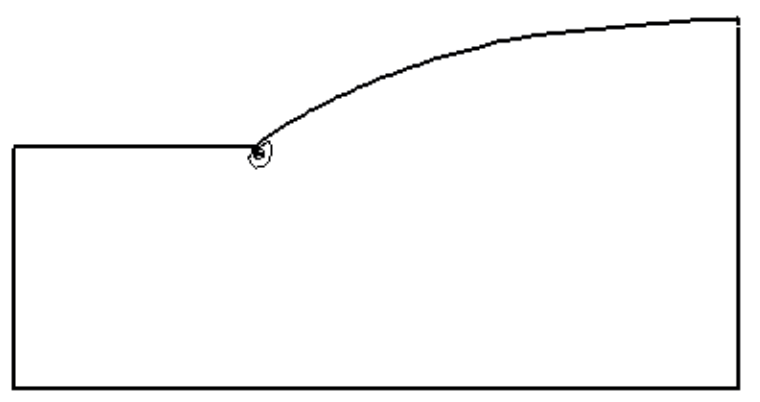

(d) $\tau_{\mathrm{rz}}$

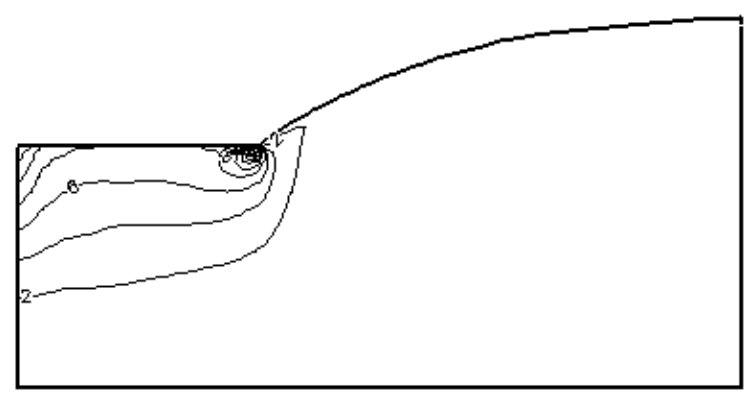

(e) $\tau_{z z}$

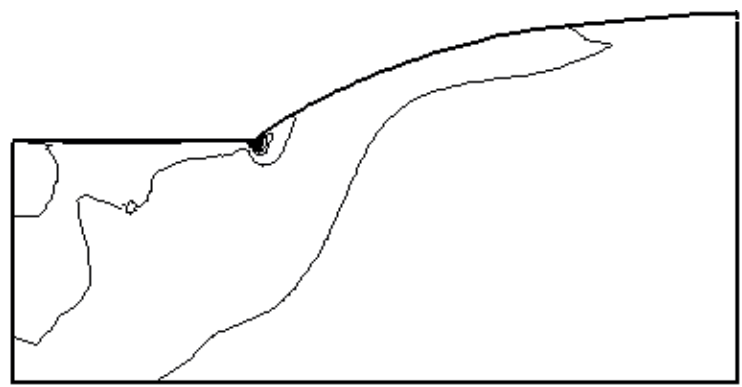

(f) $\tau_{\text {rT }}$

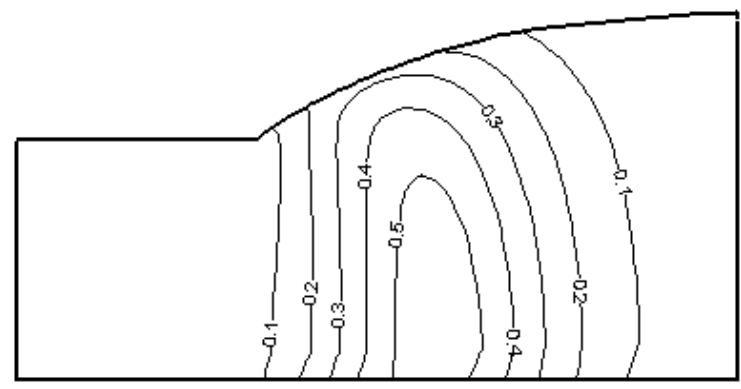

(g) $\tau_{\theta \theta}$

Figure 12: Line contour of Oldroyd-B fluid at $W e=2$. (continued)

\section{Conclusions}

The simulation of die-swell problem was evaluated with efficiency procedure of feedback Semi-implicit Taylor Galerkin Finite Element Method whilst the algorithm without feedback can execute only half value of restricted standard Weisenberg number for Oldroyd-B fluid. By means of feedback pressuredriven velocity flow, all stresses, swelling ratio and pressure are enlarged when $W e$ is increased to the upper limit of two. The well solution is close to real problem that is the reason from the re-force of velocity and pressure fields at the inlet boundary in order to improve the swelling ratio that is slightly different to the theoretical prediction. The swelling line of free surface has reached steadiness faster than non-treatment method that took further time steps and its solution of fixing initial condition closely matches analytical adjustment.

\section{Acknowledgments}

The authors would like to thank the scholarship from National Science and Technology Development Agency (NSTDA), Thailand to support PhD degree and Advanced Virtual and Intelligence Computing (AVIC) at the Department of Mathematics and Computer Science, Faculty of Science, Chulalongkorn University to sustain the advanced computer machinery.

\section{References}

[1] J. Vlachopoulos, M. Horie, and S. Lidorikis, "An Evaluation of Expressions Prediction Die Swell," Trans. Soc. Rheol., vol. 16(4), pp. 669-685, 1972.

[2] S. Richardson, "A Stick-Slip Problem Relatived to the Motion of a Free Jet at Low Reynolds Numbers," in Proc. Camb. Phil. Soc., 1970, vol. 67, pp. 477-489.

[3] M. Okabe, "Fundamental Theory of the SemiRadial Singularity Mapping with Applications to Fracture Mechanics," Comp. Meth. App. Mech. Eng., vol. 26, pp. 53-73, 1981.

[4] V. Ngamaramvaranggul and S. Thenissara, "The Contraction Point for Phan-Thien/Tanner Model of Tube-Tooling Wire-coating Flow," Int. J Math. Comp Phys Quan. Eng., vol. 4(4), pp. 627-631, 2010.

[5] T. Chandrupatla and A.D. Belegundu, Introduction 
to Finite Elements in Engineering, 4th ed., Prentice Hall, 2011.

[6] M.G. Larson and F. Bengzon, The Finite Element Method: Theory, Implementation, and Application, Springer Berlin Heidelberg, vol. 10, 2013.

[7] O.C. Zienkiewicz, R.L. Taylor, and J.Z. Zhu, The Finite Element Method: Its Basis and Fundamentals, 7th ed., Butterworth-Heinemann, 2013.

[8] Aboubacar M. and Webster M.F., "A Cell-Vertex Finite Volume/Element Method on Triangles for Abrupt Contraction Viscoelastic Flows," J. NonNewtonian Fluid Mech., vol. 98, pp. 83-106, 2001.

[9] F. Belblidia, H. Matallah, B. Puangkird, and M.F. Webster, "Alternative Subcell Discretisations for Viscoelastic Flow: Stress Interpolation," J. Non-Newtonian Fluid Mech., vol. 146, pp. 59-78, 2007.

[10] I.J. Keshtibana, B. Puangkird, H. TamaddonJahromia, and M.F. Webster, "Generalised Approach for Transient Computation of Start-Up Pressure-Driven Viscoelastic Flow," J. NonNewtonian Fluid Mech., vol. 151, pp. 2-20, 2008.

[11] B. Puangkird, F. Belblidia, and M.F. Webster, "Numerical Simulation of Viscoelastic Fluids in Cross-Slot Devices," J. Non-Newtonian Fluid Mech., vol. 162, pp. 1-20, 2009.

[12] D.B. Ingham and M.A. Kelmanson, Boundary Integra Equation Analyses of Singular Potential and Biharmonic Problems, Spinger-Verlag, Berlin, 1984

[13] R.E. Nickell, R.I. Tanner, and B. Caswell, "The Solution of Viscous Incompressible Jet and FreeSurface Flows Using Finite-Element Methods," J. Fluid Mech., vol. 65, pp. 189-206, 1974.

[14] M.J. Crochet and R. Keunings, "Die Swell of a Maxwell Fluid Numerical Prediction," J. NonNewtonian Fluid Mech., vol. 7, pp. 199-212, 1980.

[15] W.J. Silliman and L.E.Scriven, "Separating Flow near a Static Contact Line: Slip at a Wall and Shape of a Free Surface," J. Comp. Phys., vol. 34, pp. 287-313, 1980.

[16] N. Phan-Thien, "Influence of Wall Slip on Extrudate Swell: a Boundary Element Investigation," J. Non-Newtonian Fluid Mech., vol. 26, pp. 327-340, 1988.
[17] A. Karagiannis, A.N. Hrymak, and J. Vlachopoulos, "Three-dimensional nonisothermal extrusion flows," Rheol. Acta, vol. 28, pp. 121-133, 1989.

[18] N. Thongjub, B. Puangkird, and V. Ngamaramvaranggul, "Simulation of slip effects with 4:1 contraction flow for Oldroyd-B fluid," AIJSTPME, vol. 6(3), pp. 19-28, 2013.

[19] V. Ngamaramvaranggul and M.F. Webster, "Viscoelastic Simulation of Stick-slip and Dieswell Flows," Int. J. Num. Meth. Fluids., vol. 36, pp. 539-595, 2001.

[20] V. Ngamaramvaranggul and N. Thongjub, "Newtonian fluid through the abrupt 4:1 contraction flow of rounded corner geometry with feedback pressure-driven velocity flow," Int. J. Inf. Tech. Comp. Sc., vol. 15(2), pp. 1-10, 2014.

[21] R.I. Tanner, "A theory of die-swell," J. Polymer. Sci. Part A, vol. 8(2), pp. 2067-2078, 1970.

[22] B. Caswell and M. Viriyayuthakorn, "Finite element simulation of die swell for a maxwell fluid," J. Non-Newtonian Fluid Mech., vol. 12, pp. 13-29, 1983.

[23] C.W. Butler and M.B.Bush, "Extrudate Swell in some Dilute Elastic Solution," Rheol. Acta, vol. 28, pp. 294-301, 1989.

[24] G.C. Georgiou, L.G. Olson, W.W. Schultz, and S. Sagan, "A Singular Finite Element for Stokes Flow: The Stick-Slip Problem," Int. J. Num. Meth. Fluids, vol. 9, pp. 1353-1367, 1989.

[25] E.O.A. Carew, P. Townsend, and M.F. Webster, "A Taylor-Petrov-Galerkin Algorithm for Viscoelastic Flow," J. Non-Newtonian Fluid Mech., vol. 50, pp. 253-287, 1993.

[26] J.R. Clermont and M. Normadin, "Numerical Simulation of Extrudate Swell for Oldroyd-B Fluid Using the Stream-Tube Analysis and a Streamline Approximation," J. Non-Newtonian Fluid Mech., vol. 50, pp.193-215. 1993.

[27] J.G. Oldroyd, "On the Formulation of Rheological Equations of State," in Proc. Roy. Soc., A200, 1950, pp. 523-541.

[28] M.J. Crochet, A.R. Davies, and K. Walters, Numerical Simulation of Non Newtonian Flow Rheology Series 1, Elsevier Science Publishers, 1984. 